\title{
OPEN Fine-grained simulations of the microenvironment of vascularized tumours
}

Received: 10 January 2019

Accepted: 1 August 2019

Published online: 12 August 2019
Thierry Fredrich $\left(\mathbb{1}{ }^{1}\right.$, Heiko Rieger ${ }^{1}$, Roberto Chignola $\mathbb{1}^{2}$ \& Edoardo Milotti $\mathbb{1}^{3}$

One of many important features of the tumour microenvironment is that it is a place of active Darwinian selection where different tumour clones become adapted to the variety of ecological niches that make up the microenvironment. These evolutionary processes turn the microenvironment into a powerful source of tumour heterogeneity and contribute to the development of drug resistance in cancer. Here, we describe a computational tool to study the ecology of the microenvironment and report results about the ecology of the tumour microenvironment and its evolutionary dynamics.

The tumour microenvironment is characterized by large chemical gradients and contains a mixture of normal and tumour cells. The presence of high gradients favours the formation of different ecological niches which can become an important source of tumour heterogeneity ${ }^{1-6}$.

The actual size of the niches is quite small, as it is mostly determined by the structure of the scaffold of capillary vessels that envelope and feed the tumour mass. This structure is highly irregular, with a chaotic blood vessel network, a missing lymphatic network, elevated acidity, poor oxygenation, and high interstitial fluid pressure. The distance between blood vessels may be as large as a few hundred $\mu \mathrm{m}$, and the regions in between them can become highly hypoxic ${ }^{4}$.

The fine-graininess and the large spatial variability are essential features in the formation of the ecological niches and they must belong to any mathematical model that aims to explain tumour heterogeneity as the result of Darwinian selection driven by the local environment. The importance of the microenvironment is widely recognized, and several researchers have tackled the problem of its description and understanding from the computational point of view, e.g. ${ }^{7-13}$, and the reviews ${ }^{14,15}$.

The studies, however, mainly focus on the biochemical, cellular and biophysical properties of the tumour microenvironment and not on its active role in providing varied evolutionary paths to genetic variants of the tumour cells. As it has already been pointed out ${ }^{16,17}$, the adaptive evolution of tumour clones (central concept of Darwinian dynamics) is driven by the formation of new environmental niches. Many practical difficulties limit the experimental study of the adaptation process, while computer simulations can shed light — albeit in a limited way - on the dynamics of many steps like the convergent evolution of different genotypes to the same phenotype, and the selective loss of specific cell functions.

Simulations of avascular solid tumours show that the microenvironment of these small cell aggregates is formed by rather homogeneous niches with smooth gradients of oxygen, of other nutrients, waste molecules and cell viability ${ }^{18}$. After the angiogenic transition, however, the microenvironment differentiates in unpredictable ways. To identify underlying processes and provide reasoning, we have developed a computational model that is a tool to understand the fine-grained features of a simplified tumour microenvironment, i.e. of a microenvironment that contains only tumour cells and blood vessels. This is a rather strong simplifying assumption, since experimental observations show that in addition to tumour cells the microenvironment contains several other important cell types such as stromal and immune cells. We wish to stress that this assumption is a necessary compromise, because no computer model can presently describe the huge biological complexity of the tumour microenvironment and capture its growth at appropriate spatial and temporal resolutions.

Our computational model combines two different simulation programs, namely, a lattice-free simulation of small avascular solid tumours and a lattice-based simulation of the blood vessels dynamics in solid tumours. As we discuss below, both models have been individually validated with experimental data, and here we provide

${ }^{1}$ Center for Biophysics \& FB Theoretical Physics, Saarland University, Saarbrücken, 66123, Germany. ${ }^{2}$ Universita degli Studi di Verona, Department of Biotechnology, Verona, 37134, Italy. ${ }^{3}$ University of Trieste, Department of Physics, Trieste, 34127, Italy. Correspondence and requests for materials should be addressed to T.F. (email: thierry@lusi. uni-sb.de) 


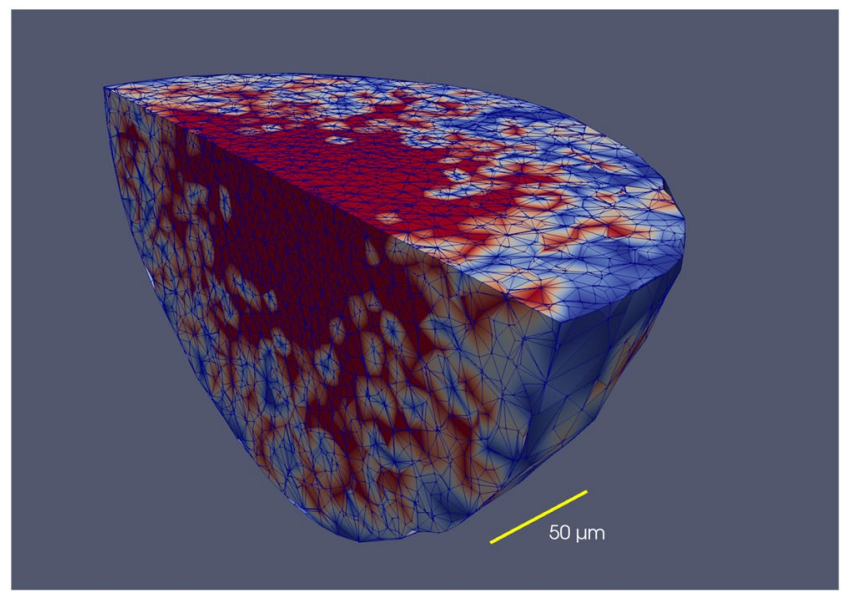

Figure 1. Distribution of cell phases in a tumour spheroid (avascular in vitro tumour) simulated with VBL. The figure shows a clipped spheroid, to display the cell phases in the inside of the spheroid. The colors represent different cell phases, and in this example it is only important to note that the necrotic regions are red. Note that there is no clear cut boundary between the proliferating part of the necrotic core. The triangulation used by VBL is also shown. This simulated tumour spheroid comprises almost 24000 cells.

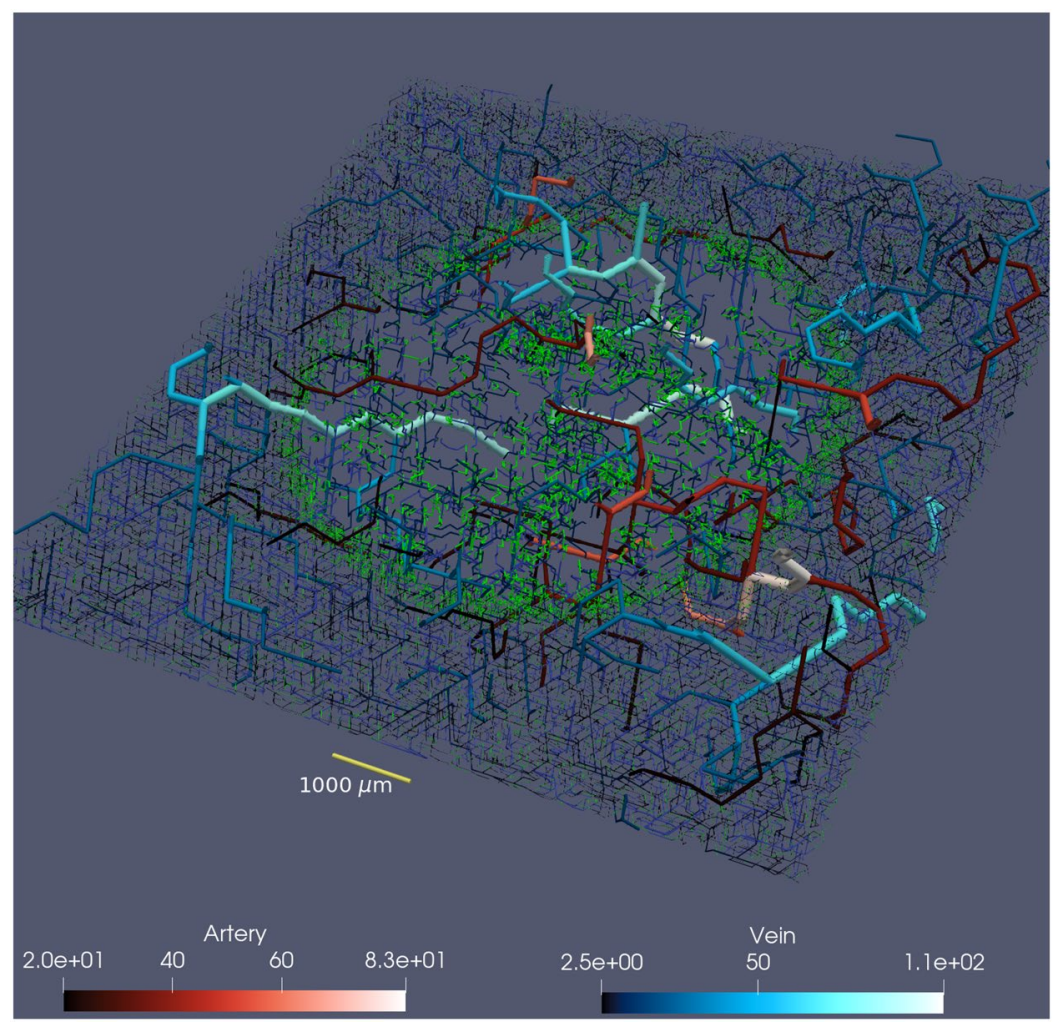

Figure 2. $500 \mu \mathrm{m}$ thick slice trough tumour vasculature of $1 \mathrm{~cm}$ lateral size. For 3D illustration we extent vessels with radius bigger than $20 \mu \mathrm{m}$ for another $750 \mu \mathrm{m}$ above and below the slice. According to the lower panels: arteries are displayed in varying reddish color and veins in varying blueish color (unit is also $\mu \mathrm{m}$ ). Capillaries and young vessel are shown in green.

a first validation of the combined model by correctly reproducing the relevant observed gradients of tumour oxygenation and other biochemical and biological features. Next, we use the combined model to explore how the tumour microenvironment takes shape in small solid tumours at the transition from the avascular to the vascular growth phases, the so-called angiogenic switch ${ }^{19}$. We follow the complex dynamics of this process in detail and in real time and show that, already at these early stages of tumour development, the diversity of environmental niches is high enough to promote adaptive evolution of tumour variants. 


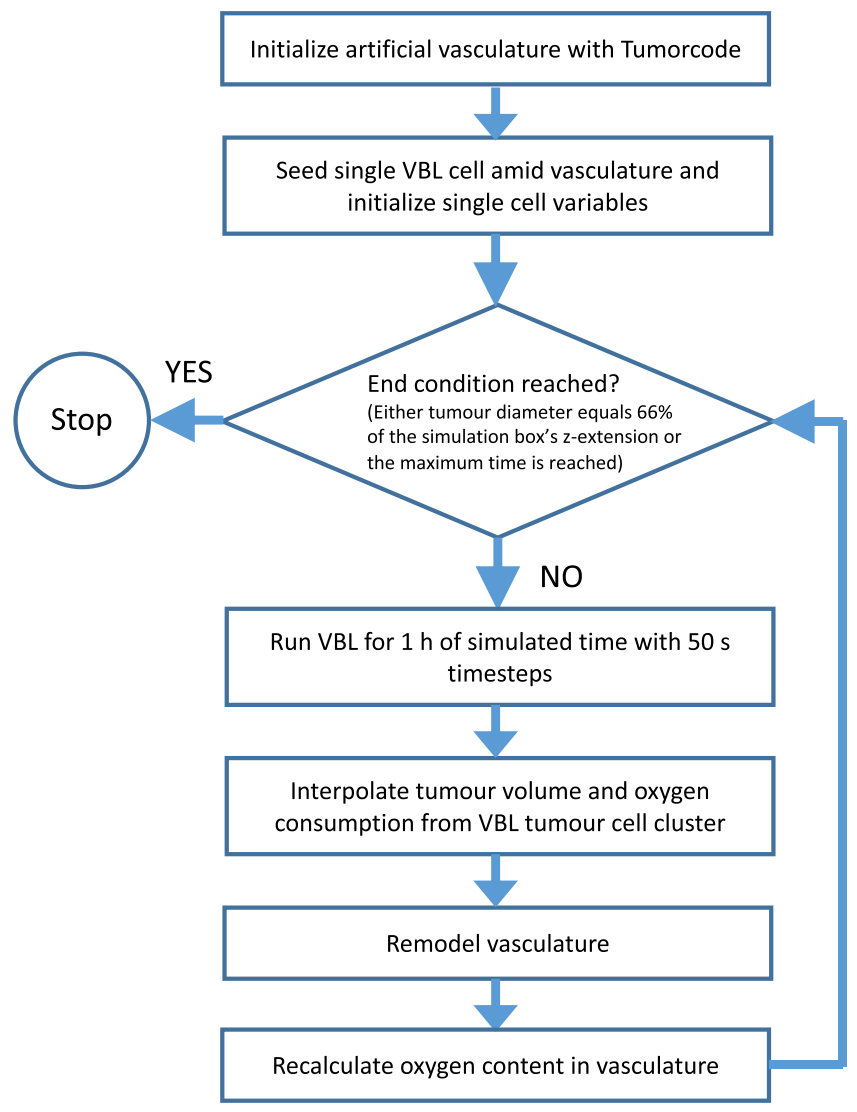

Figure 3. Flowchart of combined VBL and Tumorcode simulation.

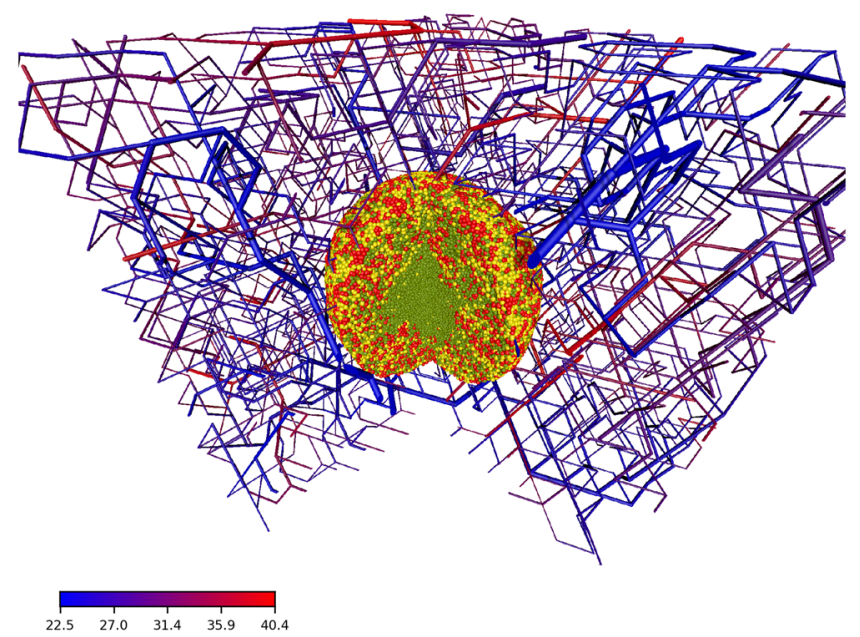

Figure 4. Artificial blood vessel network containing a tumour after 23 simulated days past the initial seeding. For visualization we removed a quarter of the system. The color code in the bottom left corresponds to the blood pressure at a given vessel segment. Cells are colored by their cell phase. Red: phases G1m and G1p; yellow: phases S, G2 and M; light green: dead cells.

\section{Brief Outline of "Virtual Biology Lab" (VBL) and Tumorcode}

In this section we give a short description of the two programs that have been merged. The present account is very brief as both programs have been described in detail in past papers (for VBL, see $e^{18,20-23}$, for Tumorcode, see $e^{24-26}$ ).

VBL. VBL is a lattice-free, cell-based program that can simulate both: the growth and proliferation of disperse cells and of more complex, avascular cell clusters (tumour spheroids). It contains a detailed simulation of the metabolic activity of each cell including discrete events at the individual cell level (for instance: mitochondrial 


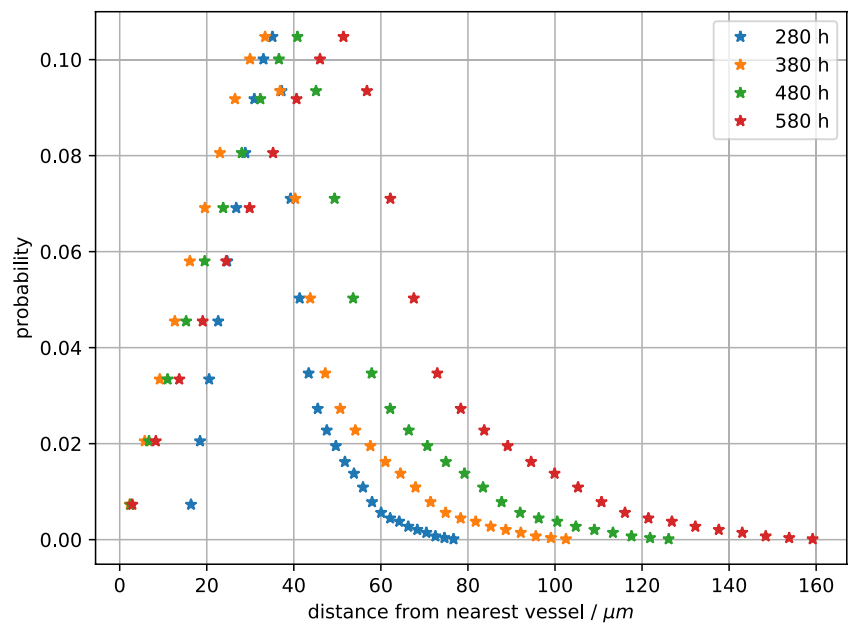

Figure 5. Empirical distribution of the distance to the closest vessel for all cells in the developing tumour. The snapshots are taken at simulated times 280 hours, 380 hours, 480 hours and 580 hours past the initial seed. Colors represent the different simulation times.

partitioning at mitosis), and has the potential to activate phenotypic differentiation. More specifically, the model has the following features:

- The model of human tumor cells includes both- the internal biochemical processes and a phenomenological description of the biomechanics of cells.

- Spatially, each cell is a two-compartment structure, the inside of the cell and the adjacent extracellular environment or intercellular space; this is important to handle simple diffusion and facilitated diffusion across the cell membrane at the same time.

- Discrete events are simulated and interleaved between successive deterministic steps; the random nature of some of them contributes to the stochasticity of the simulation as a whole.

- Each cell is characterized by its own phenotype which means that a specific set of parameters is linked to an individual cell.

- Enzyme activity is modulated both by $\mathrm{pO}_{2}$ concentration and by $\mathrm{pH}$.

- Weighting of molecular paths and cellular mechanisms is possible.

- The program simulates dispersed tumor cells and cell clusters (tumor spheroids) including the intercellular space.

- The surrounding environment is an integral part of the simulation.

The numerical methods used in VBL have been described elsewhere ${ }^{27}$, here we only mention that although the simulation is lattice-free, continuum processes are actually discretized on the continually-variable irregular lattice defined by the Delaunay triangulation ${ }^{28}$ based on the cells' centres which also defines the proximity relations among cells that are necessary to compute the cell-cell forces. We find that the computational geometry library CGAL (https://www.cgal.org) ${ }^{29}$ is an easy tool that offers necessary triangulation features amended by the possibility to calculate alpha shapes. This is necessary to define the surface or contact zone of the spheroid with the environment. It is important to note that the simulation spans many orders of magnitude in time which makes the large system of differential equations describing the deterministic part of the program extremely stiff. Therefore we solve the equations by means of implicit integration methods, that allow for comparatively large time steps (of the order of $1-10 \mathrm{~s}$ in terms of simulated time $)^{30}$.

Figure 1 shows an example of a tumour spheroid simulated by VBL. As we have shown in the past, the main metabolic, morphologic and kinetic parameters are correctly reproduced both for isolated cells and for cells grown as three- dimensional clusters ${ }^{21,27}$.

Tumorcode. While cell-cell interactions like in VBL naturally produce nearly spherical structures, modelling the growth of blood vessels moves the complexity of the simulation one step higher to the level of tissue morphogenesis. The list of models is long. For an appropriate discussion of the existing models for tumor vasculature $\mathrm{see}^{31}$.

Solid tumours grow in originally healthy tissue featuring a normal vasculature build up by a hierarchically organized arterio-venous blood vessel network that is then dynamically modified by the growing tumor. An integrative modeling approach thus has to address two issues: first, it has to find an appropriate representation of the original vasculature of the host tissue; and second, the dynamics of the given blood vessel network has to be defined, which includes the insertion of new vessels via angiogenesis as well as the removal of existing vessels (old and new) via vessel regression, and the modification of existing vessels via dilation, constriction or occlusion. Moreover, the network carries a blood flow and transports and emits oxygen, nutrients and drugs, which has to be represented, too. Various modeling approaches have been presented in the past (for a review see e.g. ${ }^{24,31,32}$ ), here 

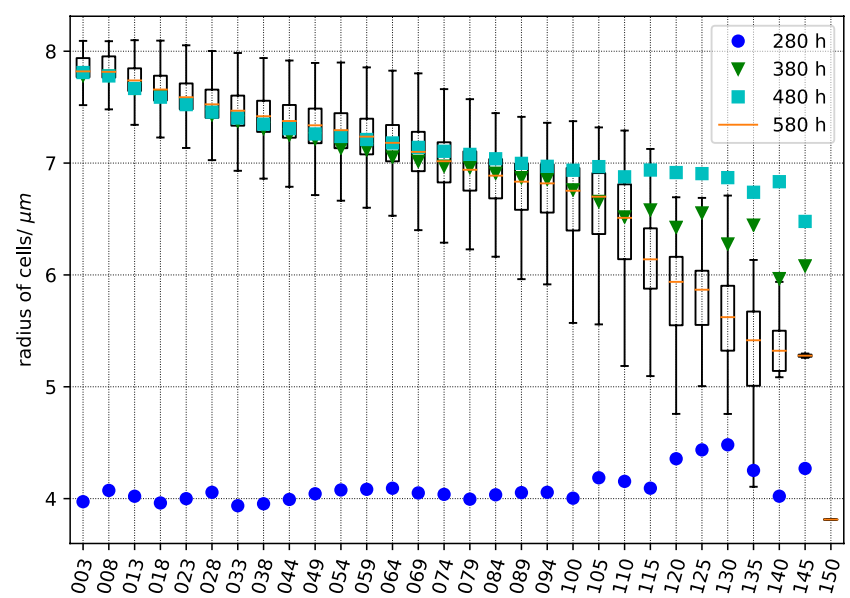

distance to nearest vessle/ $\mu \mathrm{m}$
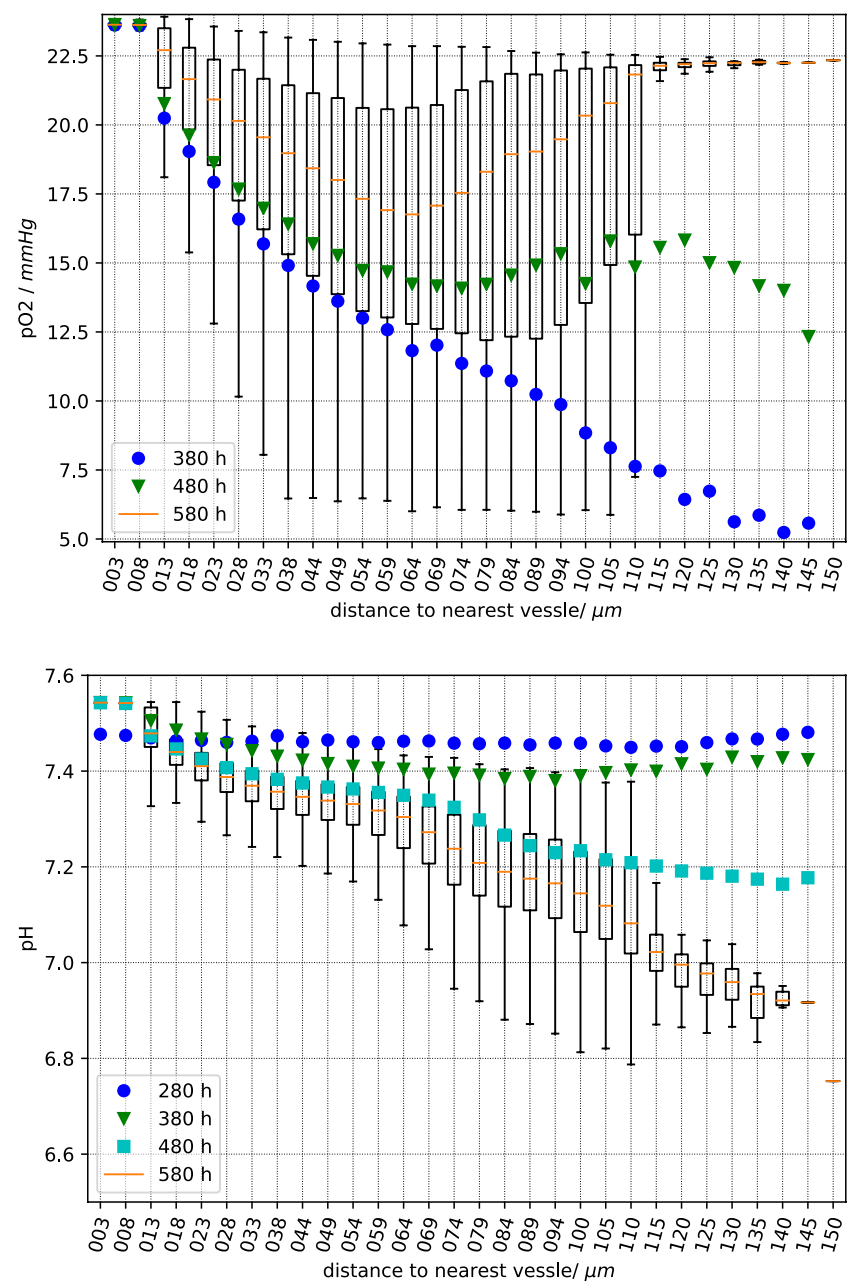

Figure 6. Sampled cell quantities vs. the distance to the nearest vessel. Top panel: cell radius, center panel: partial oxygen pressure, bottom panel: extracellular $\mathrm{pH}$. Each box plot has been obtained using the values in cells at a given distance from the nearest vessel as indicated by the abscissa. As usual, the vertical error bars ("whiskers") represent the lower and upper quartile. The data is sampled 280, 380, 480 and 580 hours past the initial seeding. For better clarity, we omit the complete boxes for the time points 280, 380 and 480 and plot only the mean value in different colors.

we use the simulation framework Tumorcode described $\mathrm{in}^{25}$, which does not only create artificial arterio-venous blood vessel networks representing the healthy initial vasculature, but includes vascular remodeling during solid tumour growth by angiogenesis, vessel dilation, regression and collapse. The created networks were tested against 

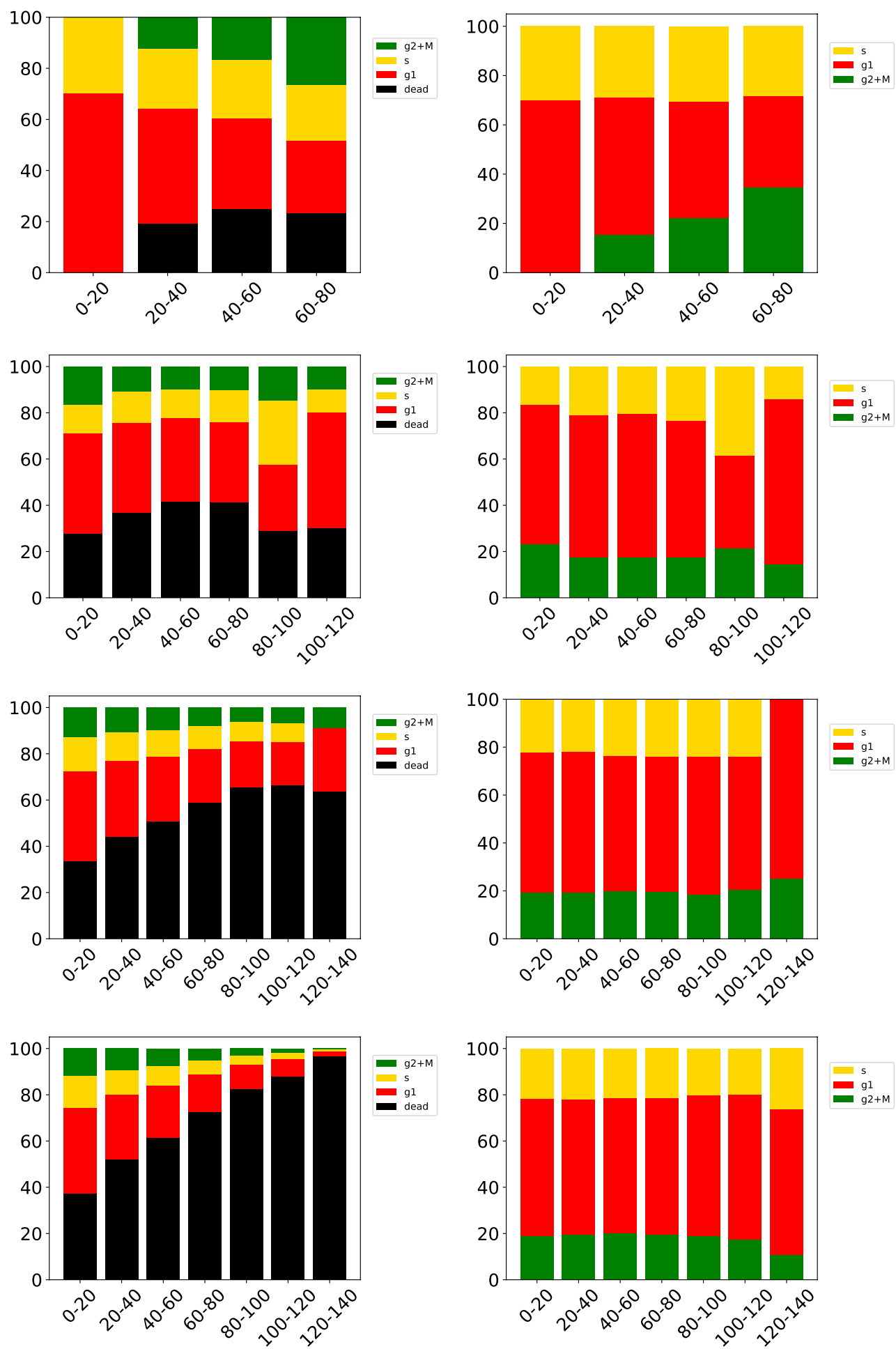

Figure 7. Distribution of the cell phases at different distances from the closest blood vessel. From top row to bottom row the data is take after $280,380,480,580$ hours past this inital seed. The left panels show the number of cells in the corresponding phase relative to the total number of current cells. G1m and G1p in red, S in yellow, G2 and M in green and dead cells in black. In the right panels we consider only alive cells. The abscissa is the distance to the nearest vessel.

experimental data and successfully reproduced: 1) morphology and flow characteristics $\left.{ }^{33,34}, 2\right)$ interstitial fluid flow and pressure ${ }^{35}$, and 3 ) tissue oxygenation ${ }^{26}$ for both, healthy and tumour tissue.

Again, here is a short list of its main features of Tumorcode: 


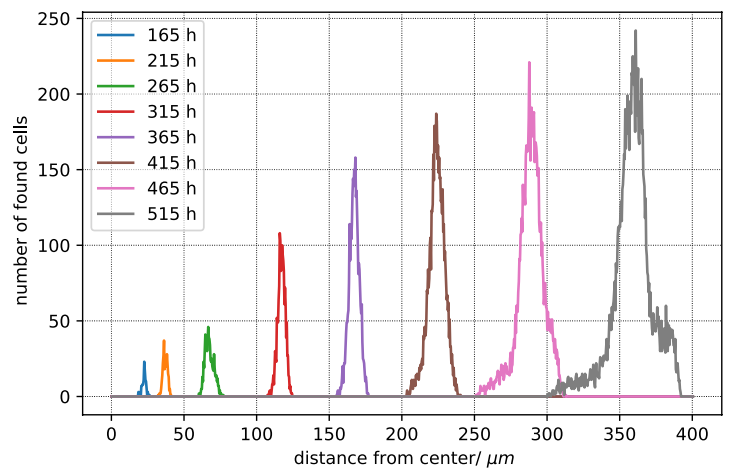

Figure 8. Distribution of distance from tumour centroid to cells in contact with the environment (normal tissue). The colors indicate the different sampling times past the inital seeding. The bin size is $30 \mu \mathrm{m}$.

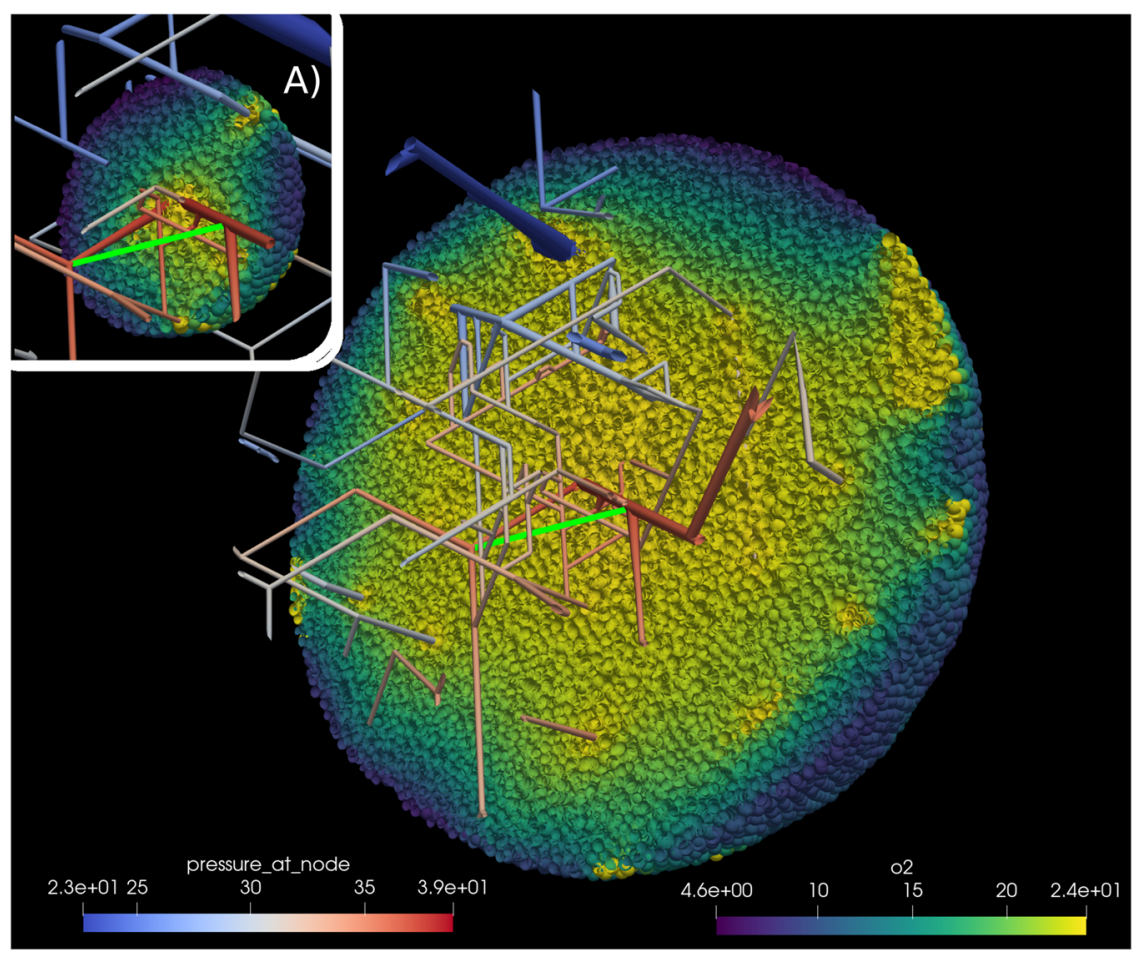

Figure 9. Sampling the partial oxygen pressure $\mathrm{pO}_{2}$ along lines between blood vessels. We sampled along the line joining two blood vessels (bright green). Inset (A) shows the simulation state 350 hours past initial seeding; the main image shows the state after 527 hours. Note the additional vessel due to angiogenessis. The scale marked "pressure_at_node" refers to the $\mathrm{pO}_{2}$ value at vessel nodes in $\mathrm{mmHg}$. The scale marked "o 2 " referes to the value of $\mathrm{pO}_{2}$ inside cells. See also Supplemental Material for more information.

- Hemodynamics includes the phase separation effect (Fåhræus-Lindqvist effect) and different rheologies.

- Concentrations in the surrounding environment are computed according to a continuum model, i.e., according to a set of partial differential equations discretized by finite elements method solved with the Trilinos C++ library (https://trilinos.org) ${ }^{36}$.

- Angiogenesis, i.e., the addition of new segments, is driven by the VGEF gradient and is partly stochastic ${ }^{24}$.

- As the tumour grows, the vasculature and the blood flows change, and the modified shear stress leads to blood vessel dilation or to blood vessel collapse $e^{24}$.

- A low local VGEF growth factor concentration produces blood vessel regression ${ }^{24}$.

- The program computes the interaction with surrounding tissues to compute the extraction of oxygen and nutrients from blood vessels.

- The program returns blood flow, oxygen concentration, metabolite concentration, etc, in blood vessels and surrounding tissues. 

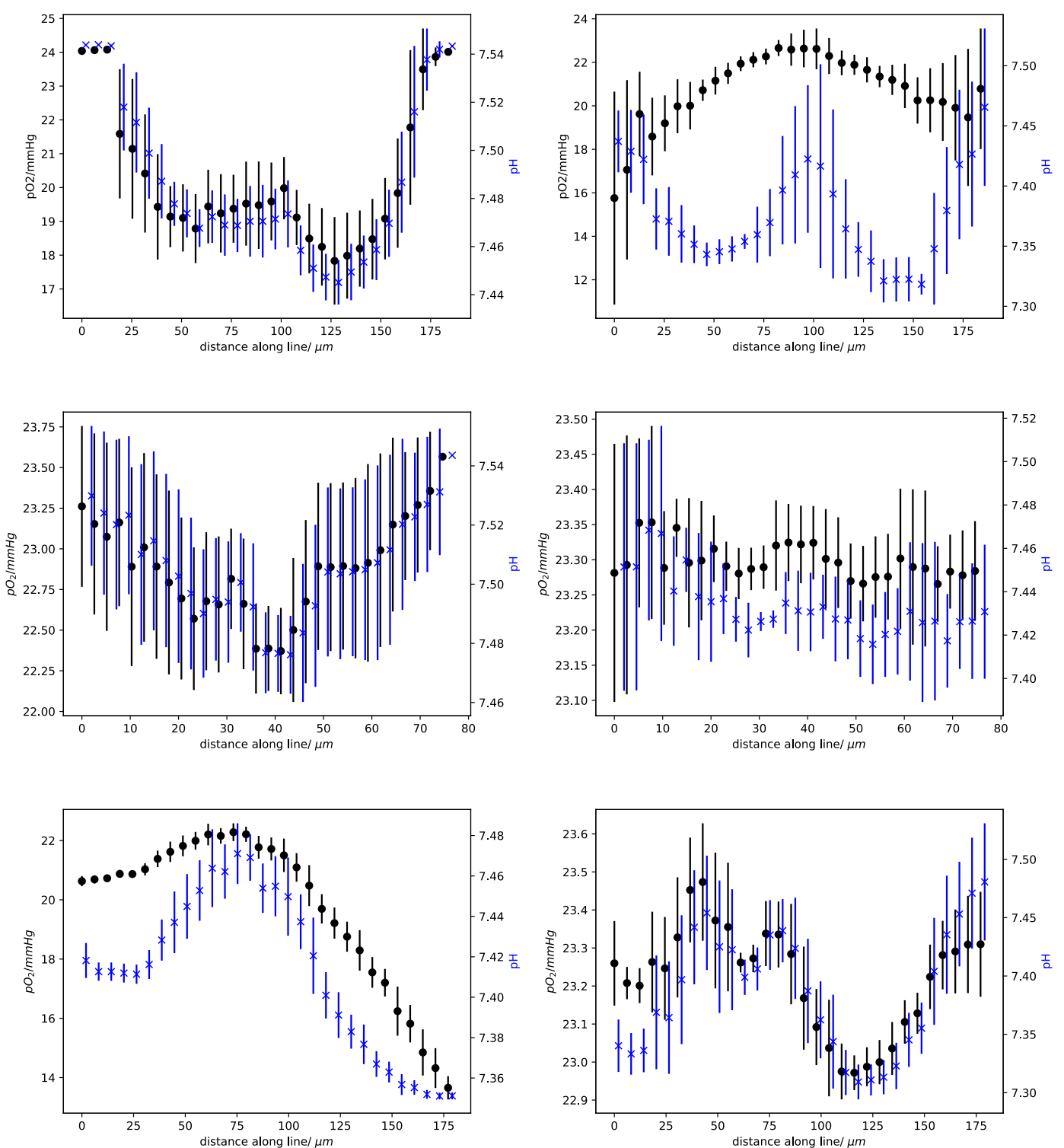

Figure 10. Data for the three measured lines. Each row corresponds to one specific line. The left column represents data taken at 350 hours of simulated time, and the right column at 495 hours past the initial tumour seeding. Each panel shows the sampled partial oxygen pressure $\left(\mathrm{pO}_{2}\right)$ in black (left hand y-axis) and the $\mathrm{pH}$ in blue (right hand y-axis). To improve visibility, the data points of $\mathrm{pO}_{2}$ and $\mathrm{pH}$ are shifted against each other by $2 \mu \mathrm{m}$. Each line was sampled by 30 points within a sphere of radius $10 \mu \mathrm{m}$. The vertical lines represent the standard deviation.

- The blood vessel hematocrit acts as source for oxygen dissolved in tissue and the tissue simultaneously consumes the oxygen. In ${ }^{26}$ we explain how the coupled set of non linear differential equations is solved to obtain the oxygen partial pressure for each blood vessel segment.

Figure 2 shows an example of a tumour vasculature simulated by Tumorcode. You can clearly distinguish the vasculature inside the tumour from the surrounding healthy tissue. Note the difference in length scale when comparing Figs 1 and 2, and the fact that the tumour is a continuous structure (governed by reaction-diffusion equation $\mathrm{s}^{25}$ ) in Tumorcode. In contrast to VBL, it cannot describe the evolutionary features of the microenvironment at the single cell level.

\section{The Merging of the Two Programs}

To merge the two programs into one, we had to match the spatial structures of the two programs. The cells are not confined to a lattice however the diffusion processes in VBL are calculated by means of a disordered Delaunay triangulation which is not permanent and has a variable size, as cells position change at each time step while the cells grow and proliferate. The situation is almost the reverse in Tumorcode where blood vessel segments belong to an FCC lattice and the continous space is discretized by a cubic lattice to solve the partial differential 


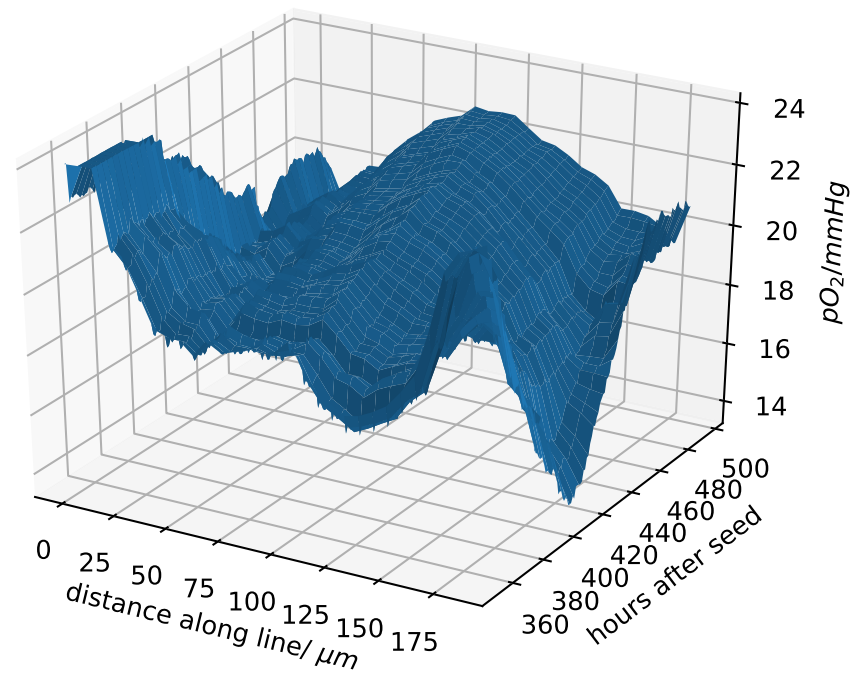

Figure 11. Change of $\mathrm{pO}_{2}$ due to the blood vessel dynamics. This data extends those in the first row of Fig. 10. In contrast to Fig. 10, we show all intermediate time points in this plot.

equations. We interpolated cell properties from the cells position to the surrounding nodes of the cubic lattice (compare Fig. 3). In particular, the Tumorcode requires an oxygen consumption field and each cell is considered as point- like source of growth factor. Once the growth factor field exceeds the threshold, the vessel remodelling of Tumorcode starts.

We estimate that the accurate calculation of any substance concentration for a given cell requires $\mathcal{O}\left(N_{\mathrm{C}} N_{\mathrm{BV}}\right)$ number of operations, where $N_{\mathrm{C}}$ is the number of cells and $N_{\mathrm{BV}}$ is the number of blood vessels segments. Since both $N_{\mathrm{C}}$ and $N_{\mathrm{BV}}$ easily exceed $10^{6}$ in the smallest-sized biologically relevant simulation runs, $\mathcal{O}\left(N_{\mathrm{C}} N_{\mathrm{BV}}\right) \sim 10^{12}$ per time step, making the simulations utterly unmanageable. To reduce the computational complexity, we take into account that only the nearest blood vessels actually contribute to the inputs and outputs of the different molecules in the vicinity of a given cell trimming the large number of cell-blood vessel pairs to $\mathcal{O}\left(N_{\mathrm{C}}\right)$. Moreover, the timesteps to follow cell biochemistry are of order 1-10 s while biological relevant timesteps for vascular remodelling are in the order of hours. Therefore we introduce two different time steps to avoid a huge number of useless sweeps over the blood vessel network (compare Fig. 3).

\section{Development of the Microenvironment in Small Solid Tumours}

With the program described before, we studied selected aspects of the tumour microenvironment. To this end, we carried out simulation runs with three different tumour seeds: 1 . at the center of a blood vessel network, 2. close to an arterial bifurcation, 3. close to a venous bifurcation. The details of each simulation are reported in the Supplemental Material. Here we display the results in a way that allows an easy comparison with measurements ${ }^{37,38}$.

As expected, the seeding is important in the very first steps of tumour growth: a seed close to blood vessels leads to fast growth and to a quick remodelling of the blood vessel network; for seeds far from blood vessels, the initial growth is slower and the subsequent initial development of the tumor microenvironment differs slightly.

Seed at the center of a blood vessel network. The computational approach provides an easy access to information that is hard to obtain with experiments and allows multiple ways of visualization (compare Fig. 4). Here we take advantage of the information about the distance to the nearest blood vessel for every cell. We start with the distribution which is a defining feature of the microenvironment, as larger distances mean less oxygen and nutrients, and therefore starvation and death for many tumour cells. In Fig. 5 we see how the distribution changes while the tumour grows. The distribution at the early stage ( 280 hours past seeding) ranges from 0 to $80 \mu \mathrm{m}$ ensuring a optimal oxygenation to all cells and gets distorted until it covers a range of up to $160 \mu \mathrm{m}$ at a later stage (580 hours past seeding) where cells not receive a sufficient input of oxygen and nutrients.

Intuitively the increase in the mean of cell-blood vessel distances leads to a decrease of available oxygen and nutrients, and to an increase of lactate ions and to a lower $\mathrm{pH}$. Since the cell-blood vessel distance is known, we can display space-time data on the distributions of cell radii, $\mathrm{pO}_{2}$ and $\mathrm{pH}$ (Fig. 6) - the data confirms the intuition. We uploaded two videos where we visualize $\mathrm{pO}_{2}$ (fredrich:po2:2018) and pH (fredrich:pH:2018) during the complete of the growth processes.

Finally, the changes in the microenvironment stretch the cell proliferation period and therefore modifies the cell cycle distribution as seen in Fig. 7 where we can follow the formation of a necrotic core. At the beginning ( 280 hours past initial seed) the relative amount of cells in the different phases varies with the distance from the nearest vessel. Surprisingly this relative amount stays almost constant after the establishment of different niches. Compare with Fig. 4 were we show the cell phase distribution in a more pictorial way. Even in such a 
small tumour we observe the initial development of necrotic regions which characterizes the development of the tumour microenvironment.

Actual tumours have irregular shapes, while the cells in VBL are not polarized, and when grown in uniform environments produce nearly spherical shapes. In the presented case the blood vessel network drives the growth in specific directions and we expect a marked deviation from sphericity already with small simulated tumours. Ideally, in a spherical tumour all cells on the surface have the same distance to the centroid. We find that the distribution of distances from the spheroid's surface to the centroid a) broadens and b) deviates from symmetric form as the tumour grows (Fig. 8) which is a clear indication of the deformation of the cell cluster and of its deviation from sphericity.

Seed close to an arterial or venous bifurcation. Helminger et al. ${ }^{38}$ measured the partial oxygen pressure and $\mathrm{pH}$ within different tumours. To compare our simulations with their experimental results, we seeded the initial tumour cell close to bifurcations, both arterial and venous, and sampled the cells along different lines (for details see Supplemental Material). Figure 9 is meant to illustrates the sampling procedure while we show data for a early and a late simulation state in Fig. 10.

It is interesting to note that the left panel in first row in Fig. 10 shows a dip in both $\mathrm{pO}_{2}$ and $\mathrm{pH}$ approximately midway between the two blood vessels. The right panel in the same row, taken at a later time, shows a maximum at roughly the same position because a new blood vessel sprouted into the intervening space. A detailed view of this dynamic change is shown in Fig. 11, where the mean of the $\mathrm{pO}_{2}$ data is plotted for all intermediate time points as the new blood vessel grows.

\section{Conclusions}

As explained in the introduction, the study of the ecology of the tumour microenvironment at the angiogenic switch is the primary motivation for the work described in this paper and for the development of the presented complex software tool. Indeed, the Figs 10 and 11 display a surprisingly large spatial and temporal variability already in a very small vascularized tumour. In particular, Fig. 11 shows a continually changing and rugged landscape: this means that the niche diversity is large, and consequently that there is a high evolutionary pressure, and a high probability that the microenvironment selects different tumour clones even in small tumours. This is important in view of the long time required for tumour growth, as it has been estimated that a tumour requires about 10 years to reach a size of $1 \mathrm{~cm}^{39}$. According to the clonal selection hypothesis new and aggressive tumour clones develop through Darwinian selection during this extended growth time $\mathrm{e}^{39,40}$. The results discussed in this paper lend further credibility to the clonal selection hypothesis, and in our future studies we plan to demonstrate the spatial and temporal variations of different clone populations in this very complex tumour microenvironment. In order to highlight common and diverse evolutionary pathways in different cancers, we also plan to explore the adaptive evolution of cell clones in different microenvironments mimicking different organs or tissue types to reproduce the distinguishing features of selected tumour types.

Finally, we note that the molecular mechanisms that promote genotypic changes in tumour clones are well known and understood, but genotypic variability is only one feature of cancer's evolutionary landscape. The other important feature is the variability of the environment that supervenes the genome and drives evolution itself, and with our computational tool we can start to explore its Darwinian dynamics ${ }^{16}$.

\section{Data Availability}

We uploaded the full simulation raw data to zenodo with following digital object identifiers: https://doi. org/10.5281/zenodo.2541688, https://doi.org/10.5281/zenodo.2541655, https://doi.org/10.5281/zenodo.2541678, https://doi.org/10.5281/zenodo.2541667.

\section{Code Availability}

All source code containing the used parameters is distributed with this article as Supplementary Material and hosted at GitHub https://github.com/thierry3000/tumorcode.

\section{References}

1. Anderson, A. R. A., Weaver, A. M., Cummings, P. T. \& Quaranta, V. Tumor morphology and phenotypic evolution driven by selective pressure from the microenvironment. Cell 127, 905-15, https://doi.org/10.1016/j.cell.2006.09.042 (2006).

2. Sprouffske, K., Merlo, L. M., Gerrish, P. J., Maley, C. C. \& Sniegowski, P. D. Cancer in Light of Experimental Evolution. Current Biology 22, R762-R771, https://doi.org/10.1016/j.cub.2012.06.065 (2012).

3. Beerenwinkel, N., Greenman, C. D. \& Lagergren, J. Computational Cancer Biology: An Evolutionary Perspective. PLoS Comput Biol 12, e1004717, https://doi.org/10.1371/journal.pcbi.1004717 (2016)

4. Yuan, Y. Spatial Heterogeneity in the Tumor Microenvironment. Cold Spring Harb Perspect Med 6, https://doi.org/10.1101/ cshperspect.a026583 (2016).

5. Basanta, D. \& Anderson, A. R. A. Homeostasis Back and Forth: An Ecoevolutionary Perspective of Cancer. Cold Spring Harb Perspect Med 7, https://doi.org/10.1101/cshperspect.a028332 (2017).

6. Gatenby, R. \& Brown, J. The Evolution and Ecology of Resistance in Cancer Therapy. Cold Spring Harb Perspect Med 8, https://doi. org/10.1101/cshperspect.a033415 (2018).

7. Shirinifard, A. et al. 3d Multi-Cell Simulation of Tumor Growth and Angiogenesis. PLoS One 4, e7190, https://doi.org/10.1371/ journal.pone.0007190 (2009).

8. Tang, L. et al. Computational Modeling of 3d Tumor Growth and Angiogenesis for Chemotherapy Evaluation. PLoS One 9, e83962, https://doi.org/10.1371/journal.pone.0083962 (2014).

9. Grogan, J. A. et al. Microvessel Chaste: An Open Library for Spatial Modeling of Vascularized Tissues. Biophysical Journal 112, 1767-1772, https://doi.org/10.1016/j.bpj.2017.03.036 (2017).

10. Xu, J., Vilanova, G. \& Gomez, H. A Mathematical Model Coupling Tumor Growth and Angiogenesis. PLoS One 11, e0149422, https://doi.org/10.1371/journal.pone.0149422 (2016). 
11. Vilanova, G., Colominas, I. \& Gomez, H. A mathematical model of tumour angiogenesis: growth, regression and regrowth. J R Soc Interface 14, https://doi.org/10.1098/rsif.2016.0918 (2017).

12. Zhao, G. et al. Three-dimensional model of metastatic tumor angiogenesis in response to anti-angiogenic factor angiostatin. J. Mech. Med. Biol. 17, 1750094, https://doi.org/10.1142/S0219519417500944 (2017).

13. Zheng, X., Wise, S. M. \& Cristini, V. Nonlinear simulation of tumor necrosis, neo-vascularization and tissue invasion via an adaptive finite-element/level-set method. Bull. Math. Biol. 67, 211, https://doi.org/10.1016/j.bulm.2004.08.001 (2005).

14. Rejniak, K. A. (ed.) Systems Biology of Tumor Microenvironment: Quantitative Modeling and Simulations. Advances in Experimental Medicine and Biology (Springer International Publishing, 2016).

15. Deisboeck, T. S., Wang, Z., Macklin, P. \& Cristini, V. Multiscale Cancer Modeling. Annual Review of Biomedical Engineering 13, 127-155, https://doi.org/10.1146/annurev-bioeng-071910-124729 (2011).

16. Gatenby, R. A., Gillies, R. J. \& Brown, J. S. Of cancer and cavefish. Nat Rev Cancer 11, 237-238 (2011).

17. Gatenby, R. A., Cunningham, J. J. \& Brown, J. S. Evolutionary triage governs fitness in driver and passenger mutations and suggests targeting never mutations. Nat Commun 5, 5499, https://doi.org/10.1038/ncomms6499 (2014).

18. Milotti, E. \& Chignola, R. Emergent properties of tumor microenvironment in a real-life model of multicell tumor spheroids. PLoS One 5, e13942 (2010).

19. Bergers, G. \& Benjamin, L. E. Tumorigenesis and the angiogenic switch. Nature Reviews Cancer 3, 401, https://doi.org/10.1038/ nrc1093 (2003)

20. Chignola, R. \& Milotti, E. Bridging the gap between the micro-and the macro-world of tumors. AIP Advances 2, 011204 (2012).

21. Chignola, R., Sega, M., Stella, S., Vyshemirsky, V. \& Milotti, E. From single-cell dynamics to scaling laws in oncology. Biophysical Reviews and Letters 9, 273-284 (2014).

22. Milotti, E., Vyshemirsky, V., Sega, M. \& Chignola, R. Interplay between distribution of live cells and growth dynamics of solid tumours. Scientific Reports 2, 990 (2012).

23. Stella, S., Chignola, R. \& Milotti, E. Competing computational approaches to reaction-diffusion equations in clusters of cells. In Journal of Physics: Conference Series, vol. 490, 012129 (IOP Publishing, 2014).

24. Rieger, H., Fredrich, T. \& Welter, M. Physics of the tumor vasculature: Theory and experiment. Eur. Phys. J. Plus 131, 31, https://doi. org/10.1140/epjp/i2016-16031-9 (2016).

25. Fredrich, T., Welter, M. \& Rieger, H. Tumorcode. Eur. Phys. J. E 41, 55, https://doi.org/10.1140/epje/i2018-11659-x (2018).

26. Welter, M., Fredrich, T., Rinneberg, H. \& Rieger, H. Computational model for tumor oxygenation applied to clinical data on breast tumor hemoglobin concentrations suggests vascular dilatation and compression. PLoS One 11, e0161267, https://doi.org/10.1371/ journal.pone.0161267 (2016).

27. Chignola, R., Del Fabbro, A., Farina, M. \& Milotti, E. Computational challenges of tumor spheroid modeling. Journal of bioinformatics and computational biology 9, 559-577 (2011).

28. De Berg, M., Van Kreveld, M., Overmars, M. \& Schwarzkopf, O. C. Computational geometry (Springer, 2000)

29. The CGAL Project. CGAL User and Reference Manual, 4.13 edn. (CGAL Editorial Board, 2018).

30. Milotti, E., Del Fabbro, A. \& Chignola, R. Numerical integration methods for large-scale biophysical simulations. Computer Physics Communications 180, 2166-2174 (2009).

31. Rieger, H. \& Welter, M. Integrative models of vascular remodeling during tumor growth. Wiley Interdiscip Rev Syst Biol Med 7, 113-29, https://doi.org/10.1002/wsbm.1295 (2015).

32. Welter, M. \& Rieger, H. Computer simulations of the tumor vasculature: Applications to interstitial fluid flow, drug delivery, and oxygen supply. Adv Exp Med Biol 936, 31-72, https://doi.org/10.1007/978-3-319-42023-3_3 (2016).

33. Welter, M., Bartha, K. \& Rieger, H. Vascular remodelling of an arterio-venous blood vessel network during solid tumour growth. Journal of Theoretical Biology 259, 405-422, https://doi.org/10.1016/j.jtbi.2009.04.005 (2009).

34. Welter, M. \& Rieger, H. Physical determinants of vascular network remodeling during tumor growth. Eur. Phys. J. E 33, 149-163, https://doi.org/10.1140/epje/i2010-10611-6 (2010).

35. Welter, M. \& Rieger, H. Interstitial fluid flow and drug delivery in vascularized tumors: a computational model. PLoS One 8, e70395, https://doi.org/10.1371/journal.pone.0070395 (2013).

36. Heroux, M. A. et al. An overview of the Trilinos project. ACM Transactions on Mathematical Software 31, 397-423, https://doi. org/10.1145/1089014.1089021 (2005).

37. Yano, S. et al. Spatial-temporal fucci imaging of each cell in a tumor demonstrates locational dependence of cell cycle dynamics and chemoresponsiveness. Cell Cycle 13, 2110-9, https://doi.org/10.4161/cc.29156 (2014)

38. Helmlinger, G., Yuan, F., Dellian, M. \& Jain, R. K. Interstitial pH and pO2 gradients in solid tumors in vivo: High-resolution measurements reveal a lack of correlation. Nature Medicine 3, 177-182, https://doi.org/10.1038/nm0297-177 (1997).

39. Talmadge, J. E. Clonal selection of metastasis within the life history of a tumor. Cancer research 67, 11471-11475 (2007).

40. Arneth, B. Comparison of Burnet's clonal selection theory with tumor cell-clone development. Theranostics 8, 3392 (2018).

\section{Acknowledgements}

The authors thank the Collaborative Research Center SFB 1027, the University of Trieste (FRA 2016), the University of Verona (RATs 2015), and the Deutscher Akademischer Austauschdienst (DAAD), for providing financial support to this project. Finally, we thank Benjamin Bogner for helping us with the computer graphics.

\section{Author Contributions}

T.F.: conducted simulations, analyzed data, drafted results part of manuscript; E.M.: suggested scientific problem, added VBL to public domain, drafted other sections; T.F. and E.M.: designed an interface for VBL and Tumorcode, optimized parameters; R.C.: helped with biological aspects and parameter tuning; H.R.: developed Tumorcode program, supervised T.F. All authors approve the content and reviewed the manuscript.

\section{Additional Information}

Supplementary information accompanies this paper at https://doi.org/10.1038/s41598-019-48252-8.

Competing Interests: The authors declare no competing interests.

Publisher's note: Springer Nature remains neutral with regard to jurisdictional claims in published maps and institutional affiliations. 
(c) (i) Open Access This article is licensed under a Creative Commons Attribution 4.0 International License, which permits use, sharing, adaptation, distribution and reproduction in any medium or format, as long as you give appropriate credit to the original author(s) and the source, provide a link to the Creative Commons license, and indicate if changes were made. The images or other third party material in this article are included in the article's Creative Commons license, unless indicated otherwise in a credit line to the material. If material is not included in the article's Creative Commons license and your intended use is not permitted by statutory regulation or exceeds the permitted use, you will need to obtain permission directly from the copyright holder. To view a copy of this license, visit http://creativecommons.org/licenses/by/4.0/.

(C) The Author(s) 2019 